\title{
Clusterorientierte Innovationsförderung - Praxis der InnoRegio FIRM
}

\author{
Michael Herzog, Kurt Erxleben
}

\section{Einleitung}

Im Jahr 1999 wurde vom Bundesministerium für Bildung und Technologie (BMBF) mit dem Programm InnoRegio ein neuartiges, wettbewerbsorientiertes Förderinstrumentarium für die Neuen Bundesländer geschaffen, das insbesondere auf die Stärkung von Kernkompetenzen in den ostdeutschen Regionen zielte und der Erhöhung der Innovationskraft und Wettbewerbsfähigkeit somit einer nachhaltigen Wertschöpfung dienen sollte. Heute wird dieses Programm unter der Dachmarke „Unternehmen Region" geführt und ist mit weiteren Programmen verbunden, die regionale Kompetenzen mit Innovationspotenzial strategisch, auf hohem technologischem Niveau und nach unternehmerischen Kriterien zu regionalen Clustern entwickeln sollen. Die Umsetzung dieses Konzepts wird weithin als einer der wenigen erfolgreichen Ansätze im „Aufbau Ost" betrachtet [1].

Die InnoRegio „FIRM“ mit ihrer Geschäftsstelle in den Räumen der Technischen Fachhochschule Wildau ist eine von 23 InnoRegios in den ostdeutschen Ländern, die sich im Jahr 2000 in einem Auswahlverfahren unter mehr als 400 Bewerbern mit ihrem Innovationskonzept durchsetzen konnte. Als Träger hatte hierzu der Verein FIRM e.V.

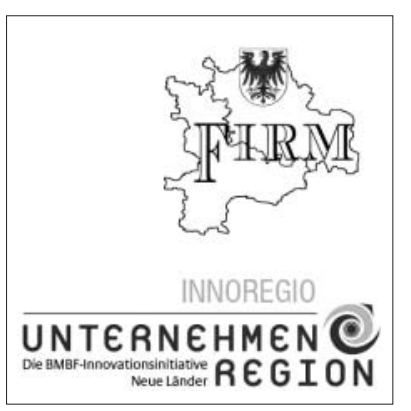

Abb. 1: InnoRegio FIRM unter der Dachmarke des BMBF „Unternehmen Region"

(Gesellschaft zur Förderung der innovativen Region Mittelostbrandenburg e. V.) das Konzept in den Landkreisen Dahme-Spreewald und Oder-Spree entwickelt. Ausgehend von Forschungserfahrungen an der Technischen Fachhochschule Wildau, dem Innovationspotential regionaler Unternehmen und der Verwaltungsexpertise der lokalen Behörden wurde ein Netzwerk konzipiert, das die Entwicklung neuer Hochleistungsmaterialien bei intelligenter Verwertung von Kunststoffabfällen in den Mittelpunkt stellt und insbesondere industrielle Reststoffe im Rahmen einer Kreislaufführung zu neuartigen Materialien umsetzt.

Heute schöpfen Akteure aus ganz Brandenburg in dem geknüpften Netzwerk ihre Innovationskraft in den Bereichen Kreislaufwirtschaft, neue Materialien und nachwachsende Rohstoffe aus.

In FIRM orientieren die Innovationen gleichzeitig sowohl auf marktfähige Produkte und auf Verfahren und Anlagen zur Herstellung der Produkte in den einzelnen Unternehmen, als auch auf die exemplarische Applikation der entwickelten Materialien in einem Produkt, das integrales
Bestandteil zahlreicher High-Tech-Anwendungen ist - ein Kryotank. Durch die Verknüpfung der wissenschaftlichen Kompetenzen der Technischen Fachhochschule Wildau und ihrer Forschungspartner in Brandenburg mit den unternehmerischen Qualitäten der lokalen Metall- und Kunststoffindustrie wird eine neue Perspektive für die Region entwickelt.

Unbestreitbare Tatsache ist, dass Unternehmen sich Innovationen nicht verordnen lassen, sie handeln selbstständig. Durch die Marktmechanismen stehen sie aber unter einem permanenten Innovationsdruck. Die Produktpalette zu erweitern oder Leistungsangebote zu modernisieren, sind alltägliche Anforderungen. Hierzu mit Hochschul- und Forschungseinrichtungen zusammenzuarbeiten, ist mittlerweile für KMU nicht ungewöhnlich. Mit anderen Unternehmen zur Erreichung übergreifender Zielstellungen zu kooperieren, erfordert mit zunehmender Wertschöpfungs- und Detailtiefe sowie komplexer Arbeitsteilung ein hohes Maß an Kommunikation und Vertrauen zur Gewährung von Einblicken in innerbetriebliche Prozesse.

Hier setzt das InnoRegio-Konzept an und moderiert die Interaktion der Partner, formt aus Wettbewerbern strategische Partnerschaften, um aus deren Stärken einen Wettbewerbsvorteil nicht nur für die unmittelbar beteiligten Akteure, sondern für die gesamte Region zu erzielen. In diesem Prozess erweist sich die Technische Fachhochschule Wildau nicht nur als Kristallisationspunkt mit den hinreichenden intellektuellen Kapazitäten zur Entwicklung von den Hochschulrahmen übergreifenden Konzepten, sondern auch als kompetenter Partner mit moderner apparativer Ausstattung zur Umsetzung von angewandten Forschungs- und Entwicklungsvorhaben mit der regionalen Wirtschaft. Die Technische Fachhochschule Wildau steht für überregional ausstrahlendes Forschungsengagement, stark nachgefragte technische Studiengänge und ausgewählte Laborkapazitäten wie das Innovations- und Gründerlabor zur Lösung von Fragestellungen im Bereich der Herstellung, Charakterisierung und Verarbeitung von neuen Materialien.

\section{Innovatives Kunststoffrecycling im Land Brandenburg}

Die Hauptquelle von Reststoffen für innovatives Kunststoffrecycling bilden Produktions- und gewerbliche Abfälle. Vorteilhafterweise haben wir es hier überwiegend mit relativ homogenen Stoffgemischen wohldefinierter Zusammensetzung zu tun; die interessierenden Fraktionen müssen also nicht in aufwändigen Sortierverfahren aus Vielstoffsyste- 
men gewonnen werden, zudem sind weitere Reinigungsbzw. Waschprozesse zur nachfolgenden Verarbeitung in der Regel nicht erforderlich. Je näher eine Verwertung an der Entstehung der Abfälle platzierbar ist, desto einfacher lässt sich durch eine Kreislaufführung die Menge des anfallenden Abfalls verringern, im Idealfall völlig vermeiden. Für thermoplastische Kunststoffe hat sich die Kreislaufführung mit Hilfe von Regranulatoren u.ä. mittlerweile im betrieblichen Alltag weitestgehend durchgesetzt, während für Duromere, sofern sie einer technischen Verwertung zugänglich sind, der Aufwand - insbesondere für Mindermengen - deutlich höher anzusetzen ist.

Die im Folgenden betrachteten Kondensationspolymere stellen mengenmäßig im sog. Consumer-Abfall nur eine untergeordnete Fraktion dar. Generell unterliegen sie jedoch einer Verwertungspflicht nach dem Kreislaufwirtschaftsgesetz, sofern es „technisch möglich und wirtschaftlich zumutbar" ist. An der Erweiterung der technischen Möglichkeiten und der Verbesserung der Wirtschaftlichkeit der Kreislaufführung von ausgesuchten Polyestern und Polyurethanen unter Weiterentwicklung des Ansatzes der externen Kreislaufführung zu anderen hochwertigen Materialien, die sich von den Ausgangsstoffen deutlich unterscheiden, wird im InnoRegio FIRM intensiv gearbeitet. Die folgenden Erörterungen reflektieren ausschließlich Verfahren jenseits einer - natürlich für alle Kunststoffe zugänglichen und in modernen Anlagen technisch ausgereiften - undifferenzierten energetischen Nutzung.

Wenn es gelingt, mittels chemischer Verfahren die Polymere so zu spalten, dass wieder molekulare Bausteine, die als Monomere oder Oligomere erneut für Polymersynthesen geeignet sind, gewonnen werden können, ist die stoffliche Verwertung nicht mehr zwangsläufig mit einem Verlust stofflicher Qualitäten verbunden - sie bleiben weitestgehend erhalten. Mit den Recyclaten aus der chemischen Umsetzung stehen dann Rohstoffe für neue Materialien zur Verfügung, deren Eigenschaften einstellbar sind und ggf. neue hochwertige Anwendungen erlauben.

Geeignete Kandidaten für solch elegante Methoden des chemischen Recyclings finden sich bei den Polyestern und Polyurethanen. Sie zeichnen sich durch carbonylische Kohlenstoffatome in den Polymerketten aus, die chemischen Umsetzungen mit Alkoholen oder Aminen zugänglich sind und somit Angriffspunkte für eine Kettenspaltung durch als Umesterung mit niedermolekularen Reaktanden zu beschreibende Reaktionen bieten. So können beispielsweise Polyurethan-Schaumstoffe durch geeignete organische Lösungsmittel (Solvent) mit Hydroxyl- oder Amin-Funktionalitäten in Oligomergemische umgewandelt werden (Solvolyse), die wieder zur Herstellung neuer Polyurethane eingesetzt werden können.

Aus Polyestern können Oligomere für die Herstellung hochwertiger Polyurethane durch eine auf deren Anforderungen eingestellte Umesterungsreaktion gewonnen werden. Durch die Glycolyse von Polyethylenterephtalat (PET) gelangt man beispielsweise zu aromatischen Polyesterpolyolen (APP), die in der Produktion von thermisch beständigen und flammgeschützten Polyurethan-Hartschaumstoffen Anwendung finden [2].

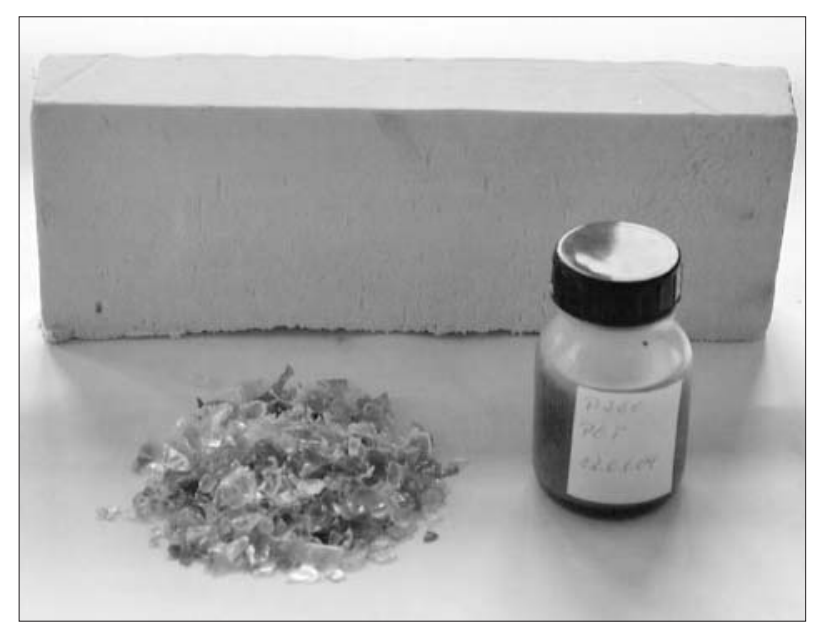

Abb. 2: PET-Abfälle, Recyclingpolyol und PUR-Hartschaumstoff

An der TFH Wildau wurden in den vergangen Jahren Verfahren zum chemischen Recycling von einerseits PET zu APP [3,5] und andererseits PUR-Schaumstoffen zu oligomeren Polyolen sowie neuartige Polyurethane daraus [4] entwickelt. Die mit den entwickelten Technologien, d.h. mit technologisch einfach zu beherrschenden Verfahren, gewonnenen Recyclate sind Gemische mehrere Polyole unterschiedlicher Struktur, deren Eigenschaften sich bereits durch die Wahl der Reaktionspartner und -parameter entsprechend des beabsichtigten weiteren Einsatzes zielgerichtet einstellen lassen. Gleichermaßen wurden anwendungsreife Technologien zur Verwendung der Recyclate in PUR-Systemen entwickelt. Die Vielfalt der gegenwärtig technisch beherrschten Anwendungen erstreckt sich hierbei von sprühfähigen Beschichtungen über Komposit-Pressplatten bis hin zu Applikationen als Hauptbestandteil in PUR-Hartschaumstoff-Formulierungen.

\section{Wesentliche Akzente von Einzelprojekten der InnoRegio Firm}

Nachdem das Grundkonzept der InnoRegio FIRM ausgearbeitet und vom BMBF mit einem Förderrahmen von ca. 5,1 Mio Euro ausgestattet worden war, galt es in der folgenden Phase, die Details der einzelnen Vorhaben auszuarbeiten und zur Bewilligungsreife zu führen. Hinter der kontinuierlich steigenden Anzahl laufender Projekte steht eine sich oft über Monate hinziehende Phase der Projektentwicklung, deren höchste Intensität im Jahr 2004 erreicht wurde.

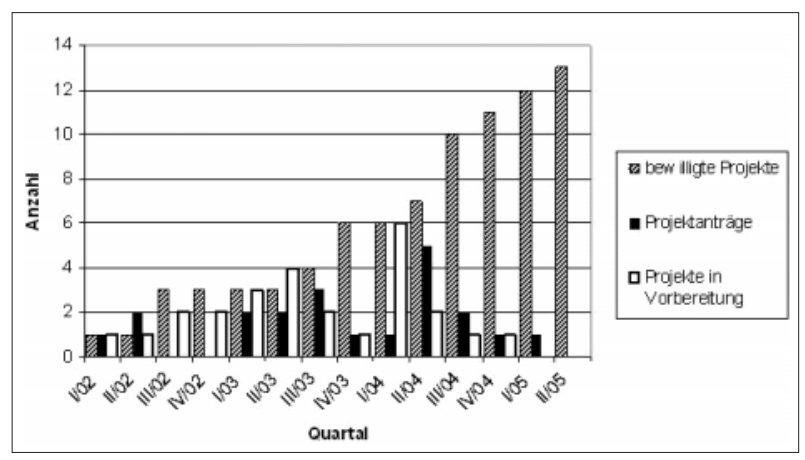

Abb. 3: Dynamik der Projektentwicklung im InnoRegio FIRM 
In der Projektbearbeitung wird im Jahr 2005 die höchste Aktivität erreicht. Von den Unternehmen werden hierzu nicht unbeträchtliche Eigenmittel für die Finanzierung ihrer Projekte aufgebracht. Auch die Planung für die in Anspruch genommenen Fördermittel erreicht im Jahr 2005 sein Maximum. Verursacht durch den Abrechnungsmodus der Unternehmen gegenüber dem BMBF erfolgt der Mittelabfluss um ein Quartal versetzt zum Leistungszeitraum.

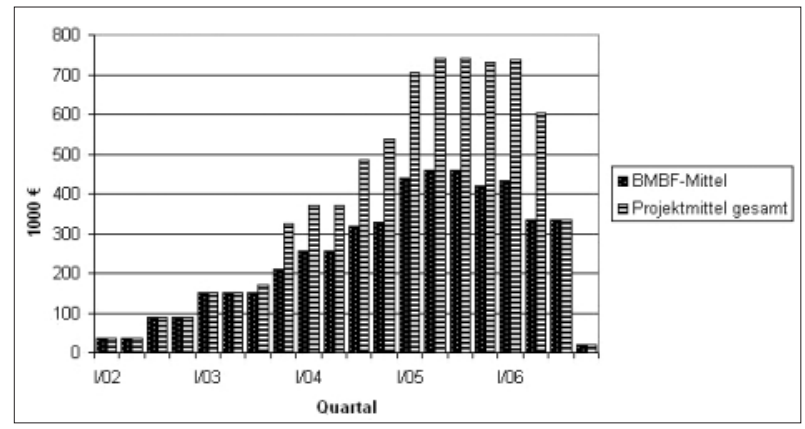

Abb. 4: Eingesetzte Projektmittel insgesamt und Mittelabfluss der BMBF-Förderung

Zentrales Steuerungselement und Koordinierungsstelle für das Netzwerk der InnoRegio FIRM ist die an der TFH Wildau beheimatete Geschäftsstelle InnoRegio FIRM [6]. Als Träger dieses Teilprojekts (TP 1) fungiert der FIRM e.V. Königs Wusterhausen.

Mit dem Studienschwerpunkt Materialtechnologie (TP 2) an der TFH Wildau wird die Entwicklung von neuen, modular aufgebauten Lehrangeboten mit starker Orientierung auf Neue Materialien vorangetrieben sowie entsprechend des Fachkräftebedarfs der regionalen Wirtschaft ausgerichtet.

Als Basistechnologie werden im Rahmen des Teilprojekts 3 (TP 3) an der TFH Wildau die Grundlagen für eine Anlage zur kontinuierlichen Herstellung von PET-Recyclingpolyolen entwickelt [2]. Die TSA GmbH, Großräschen betreibt im TP 4 die Entwicklung und Konstruktion eines kontinuierlichen Versuchsreaktors zur Herstellung von Recyclatpolyolen aus PUR-Weichschaumstoffen. Beide Recyclattypen dienen in den Technologie- und Materialentwicklungen weiterer Teilprojekte als Ausgangsstoffe für unmittelbar anwendbare technische Produkte.

Im Teilprojekt 5 wird ein anderer Weg des stofflichen Recyclings verfolgt, bei dem Polyesterabfälle zu eigenverstärkten Kunststoffen bzw. Kunststoffhalbzeugen verarbeitet werden sollen. Hierzu entwickelt das Traditionsunternehmen hermann römmler-kunststofftechnik KG, Rehfelde ein neuartiges Spritzgiessverfahren für strukturierte PBT-Formkörper, während durch die Kombination eines Press- und Reckverfahrens bei der Gloger+Birke GmbH, Jacobsdorf Platten erhöhter Festigkeit erzeugt werden sollen.

Mit der Entwicklung von Beschichtungen zur Innenund Außenbeschichtung von Flüssigkeitstanks auf der Basis von Recyclingpolyolen nutzt die fenotec $\mathrm{GmbH}$, Beelitz im TP 6 in großem Umfang Ausgangsstoffe aus der Kreislaufführung von PUR, vorzugsweise auf der Basis der Ergebnisse von TP 4.

Einen weiteren Aspekt verfolgt die Greibo-Chemie $\mathrm{GmbH}$, Velten mit der Entwicklung von gekoppelten Verbindungen aus nativen Ölen oder deren Derivaten und eines Verfahrens zur Herstellung von Flammschutzmitteln aus diesen Verbindungen. Im TP 7 werden nachwachsende Rohstoffe zu Zuschlagstoffen für die im Rahmen des Netzwerks entwickelten neuen Materialien umgesetzt.

In einem Verbundprojekt (TP 8) widmen sich die TFH Wildau und die ReMetall GmbH, Drochow der Entwicklung von kältebeständigen Distanzkörpern aus leichten keramischen Massen. Zur Erzeugung von Keramikschäumen kommen hierbei sowohl Recyclingpolyole aus o.g. Prozessen, als auch Material aus dem Recycling von AbgasKatalysatoren zum Einsatz.

In TP 9 verfolgt die Dreskon GmbH, Radebeul einen aussichtsreichen Ansatz zur Entwicklung der Isolationsschichten für einen Flüssiggastank aus tieftemperaturstabilen PURHartschaumstoffen auf der Basis von Recyclingpolyolen.

Mit dem TP 10 beabsichtigt die Europarts Drochow $\mathrm{GmbH}$ die Entwicklung eines Transport- und Lagerbehälters für Flüssiggase, speziell eines Kryotanks. Ein derartiger Tank, der in zahlreichen Zukunftstechnologien integraler Bestandteil komplexer Verfahrenskonzepte ist, dient in Detaillösungen der Demonstration der Leistungsfähigkeit der im Netzwerk bzw. in den Teilprojekten erarbeiteten Materialien.

Eine weitere Nutzung nachwachsender Rohstoffe für Anwendungen in der Kunststoffindustrie wird in TP 11 mit der Entwicklung von hydroxylgruppenfunktionellen Vernetzern durch elektrochemische Oxidation nativer Öle durch die HAWO GmbH, Hohenbucko angestrebt.

Mit dem Ziel der Kostenreduktion in einem etablierten industriellen Verfahren durch den Einsatz von Recyclingpolyolen betreibt die platec Elsterwerda GmbH das TP 12 zur Entwicklung eines neuen Verfahrens zur Herstellung von Plattenmaterial aus verschiedenen Polyurethanabfällen.

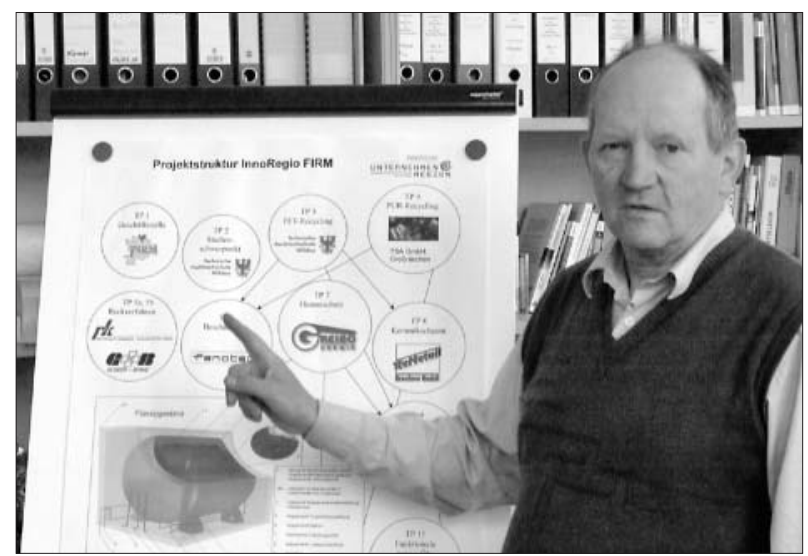

Abb. 5: Erläuterung der Projektstruktur in der Geschäftsstelle InnoRegio FIRM an der TFH Wildau

Die Technische Fachhochschule Wildau ist an allen Teilprojekten neben der Projektentwicklung auch an der fachlichen Diskussion der Ergebnisse der Vorhabensbearbeitung sowie mit der Erbringung von wissenschaftlichen Dienstleistungen beteiligt.

\section{Ausblick}

Mit seiner auf die globalen Märkte gerichteten internationalen Ausrichtung spiegelt InnoRegio FIRM die Unternehmen-Region-Philosophie des BMBF in optimaler Weise wider. 
Diese Entwicklung kann als Modell für die nachhaltige Entwicklung bislang strukturell benachteiligter Regionen dienen, mit dem erprobt wird, wie rein ökonomisch bestimmtes Handeln, erweitert um Erfolgsfaktoren wie Fairness [7] und dem erfolgreichen Agieren in Netzwerken, zukunftsfähige Regionen mit klarem Kompetenzprofil wachsen lässt.

Die wissenschaftliche Begleitforschung des InnoRegioProzesses konnte die Wirksamkeit der eingesetzten Mittel zur Stärkung der Wettbewerbsfähigkeit und Leistungskraft von Unternehmen im InnoRegio-Programm eindrucksvoll empirisch belegen [8].

Mit weltweiten Partnern zusammenzuarbeiten ist im Zeitalter der modernen Telekommunikation und des Internets für jede Firma möglich. Für die Formierung eines Clusters sind neben der räumlichen Nähe der Partner (um gemeinsames Wissen und Ressourcen sinnvoll teilen zu können) auch Verbindungen und gemeinsame Ziele notwendig. Als wichtigste Kriterien gelten die Interaktion der Partner und das Erreichen einer „kritischen Masse“, um den Standort und den regionalen Kontext zu einer Stärke, in einen Wettbewerbsvorteil für die Unternehmen und einen Nutzen für potentielle Kunden umzumünzen [9].

InnoRegio FIRM findet sich im Land Brandenburg in den Branchen-Kompetenzfeldern Kunststoffe und Umweltwirtschaft sowie mit den entwickelten Neuen Materialien im Cluster Luft- und Raumfahrt [10] wieder.

Region wird jedoch zunehmend nicht nur geographisch verstanden, sondern als Raum zur Generierung und Durchsetzung von Innovationen. Insofern versteht sich die Region FIRM als eine innovative Region mit dem Schwerpunkt auf Materialtechnologien mit Anwendungen bis hin zur Luftfahrt.

Zum Schluss greifen wir ein etwas heiteres Denkbild von Peter Sloterdijk auf, der die Netzmetaphorik als Ausdruck einer stark reduktiven Geometrie, als bloß ein- oder zweidimensionale Form korrigieren möchte - und zwar mit dem Ausdruck „Schaum“. Während im Netzwerkmodell die einzelnen Punkte kein Volumen besitzen, öffnet das Bild vom Schaum den Ausblick auf eine Theorie der Vielfalt, auf eine multifokale Entfaltung, die den einzelnen Knotenpunkten im Netz erlaubt in ihrer relativen Autonomie gegenüber dem übrigen Netz besser respektiert zu werden [11].

Schaumstoffe sind im InnoRegio FIRM sowohl Ausgangsstoff (PUR-Schaumstoffe) als auch Ziel neuer Materialentwicklungen (PUR-Hartschaum und Keramikschaum). Mit der Begriffswahl „Schaum“, so ist sich auch Sloterdijk bewusst, ist auch ein Charaktertest hinsichtlich der Versuchung zu billiger Ideenmechanik a la "Schaumschlägerei“ verbunden.

Dem widerstehen wir respektvoll sowohl in der Aufweitung von Netzwerkkonzepten wie auch unter dem Aspekt unserer Materialentwicklungen, die miteinander partizipieren und voneinander profitieren.

\section{Literatur/Anmerkungen}

[1] Innovationskonferenz „Aufbau Ost“ des BDI, BMBF und BWA, 31.03.2005 im Haus der Deutschen Wirtschaft, Berlin

[2] R. Langenstraßen, S. Fulev, A. Apel, B. Gebert, D. Lehmann, G. Behrendt: Entwicklung der Grundlagen für eine Laboranlage zur kontinuierlichen Herstellung von PET-Recyclingpolyolen, Wiss. Beitr. Techn. Fachhochschule Wildau 2004, S. 34

[3] G. Behrendt, M. Pohl: Polyester-Polymerpolyole für Polyurethane und Verfahren zu ihrer Herstellung, DE 19915125 C2 (2001)

[4] G. Behrendt, M. Pohl: Verfahren zur Herstellung von Polyurethanen, DE 19917934 (1999)

[5] G. Behrendt, M. Pohl: Verfahren zur Herstellung von Polyolen sowie Polyole, DE 19917932 (1999)

[6] H. Kupper: Zur Kunst der Projektsteuerung: Qualifikation und Aufgaben eines Projektleiters, Oldenbourg Verlag München Wien, 2001

[7] A. Ockenfels: Frankfurter Allgemeine Sonntagszeitung, 27.03. 2005, S. 39

[8] InfoBrief der wissenschaftlichen Begleitung der InnoRegio Initiative, Nr. 10 DIW Berlin (2004)

[9] C. Ketel: Motoren für die Region, Unternehmen Region 2/2004, S. 12, Hrg. BMBF

[10] Pressemitteilung des Ministerium für Wirtschaft des Landes Brandenburg vom 02.03.2005 zur Neuausrichtung der Wirtschaftsförderung

[11] F. Hartmann, K. Taschwer im Gespräch mit P. Sloterdijk über sein Buch „Sphären, Bd. III Schäume“, Surkamp 2004

\section{Autoren}

Dr. rer. nat. Michael Herzog

Technische Fachhochschule Wildau

Fachbereich Ingenieurwesen/Wirtschaftsingenieurwesen Geschäftsstelle InnoRegio FIRM

Tel. + 493375 508-332

mherzog@igw.tfh-wildau.de

Dr. rer. agr. Kurt Erxleben

Geschäftsstelle InnoRegio FIRM an der TFH Wildau

FIRM e.V. Königs Wusterhausen

Tel. + 493375 508-354

k.erxleben@firm-ev.de 